\title{
Chaotic behavior of ionospheric scintillation: Modeling and observations
}

\author{
A. W. Wernik \\ Space Research Centre, Polish Academy of Sciences, Warsaw \\ K. C. Yeh \\ Department of Electrical and Computer Engineering, University of Illinois at Urbana-Champaign, Urbana
}

\begin{abstract}
The chaotic, but nondeterministic, structure of the ionosphere and radio wave amplitude and phase scintillation measured on the ground have been simulated using the phase screen model with the power law spectrum of random Gaussian phase fluctuations. Comparison of the information dimension calculated for phase fluctuations on a screen with that for amplitude scintillation at the receiver shows that the original chaotic structure in the ionosphere is completely masked by the propagation effects. Hence the ionospheric turbulence attractor (if it exists) cannot be reconstructed from amplitude scintillation data. On the other hand, measured phase scintillation data adequately reproduce the assumed chaotic structure in the ionosphere. Results of the attractor reconstruction for amplitude scintillation observed at high latitudes show an excellent agreement with our simulation.
\end{abstract}

\section{Introduction}

In recent years the concepts of chaos and nonlinear dynamics have found application in studying the behavior of various complex systems. Their attractiveness stems from the fact that they provide a relatively simple statistical description of the system. Although the use of the nonlinear dynamics approach does not tell us about the physical nature of a system, the theoretical model of the system should aim in reproducing the results of nonlinear analysis.

It is known that ionospheric plasma turbulence causes scintillation of transionospheric radio signals. An important question is what can we learn about the irregular structure from scintillation measurements. The answer to this question is provided by the scintillation theory which relates the measured statistical parameters of scintillation and statistics of irregularities [Yeh and Liu, 1982]. It has been found, for instance, that the phase and amplitude scintillation spectra can be used to deduce the form of the power spectrum of electron density fluctuations. However, the power spectrum does not describe unambiguously the turbulent nature of

Copyright 1994 by the American Geophysical Union.

Paper number 93RS01828.

0048-6604/94/93RS-01828\$08.00 the ionosphere [Costa and Kelley, 1978; Bhattacharyya, 1990] since it gives only the power of Fourier components, while the information about the phase coherence between components is missing. An alternate description of ionospheric turbulence can be inferred using the chaotic system concept. It gives an independent measure known as fractal or Hausdorff-Besicovich dimension characterizing the "degree of chaos" or "degree of determinism" which is present in the experimental data. In other words, the fractal dimension provides some measure of the number of degrees of freedom or independent parameters describing the system under study. It is based on the rough notion that the fractal dimension increases with the complexity of the system. More precisely, the fractal dimension is the dimension of the strange attractor, a certain domain of phase space toward which converge all trajectories of the chaotic system ([Bergé et al., 1987] gives an excellent introduction to the chaos theory). Alternative dimensions, characterizing the attractor, include the information dimension and the correlation dimension. In most cases they are equal to the fractal dimension.

The chaotic behavior of ionospheric electron density fluctuations resulting from the interchange instabilities has been investigated by $\mathrm{Huba}$ et al. [1985] and Hassam et al. [1986]. In the first paper it has been shown that for a three-mode system the 
nonlinear equations describing the Rayleigh-Taylor and $\mathbf{E} \times \mathbf{B}$ gradient drift instabilities reduce to equations which describe the so-called Lorenz attractor for Rayleigh-Benard instability, a typical example of the chaotic system. In the development of chaotic behavior the ion inertia plays a critical role in that if it is neglected, as below $500 \mathrm{~km}$ in the ionosphere, the three-mode system does not exhibit chaos, and a stable convection results. Hassam et al. [1986] have shown, however, that this is not so when many mode coupling is allowed. At the same time, in the inertial regime, for which the threemode theory predicts chaos, the large-scale turbulence cells do not show chaotic behavior.

An attempt to obtain information about the chaotic behavior of ionospheric plasma density has been made by Bhattacharyya [1990] who has analyzed phase and amplitude scintillation data. Her main conclusions are (1) ionospheric turbulence is generally low dimensional; (2) as the strength of amplitude scintillation increases, its information dimension increases, possibly due to the "focusing" of the incident wave. Thus a higher scintillation intensity does not necessarily imply higher dimensionality of the ionospheric plasma turbulence.

In this paper we will relate the chaotic behavior of ionospheric turbulence and scintillation using numerical modeling of scintillation and compare the results with observations made at high latitudes.

\section{Numerical Mode of Scintillation}

To model the amplitude scintillation resulting from wave propagation through the irregular ionosphere, we can apply the method described, for instance, by Knepp [1982] and Rino and Owen [1984]. In this method the phase screen model is used in which the irregular ionosphere affects only the phase of the wave. The amplitude fluctuations develop during propagation between the screen and the ground as a result of phase mixing. If the dimension of the first Fresnel zone is much smaller than the outer scale of the phase fluctuations on the screen, the wave field on the ground can be computed using the Kirchhoff's diffraction formula [Yeh and Liu, 1982]:

$$
u(x, z)=\frac{i k A_{0}}{2 \pi z} \int \exp \left\{-i\left[\phi\left(x^{\prime}\right)+(k / 2 z)\left(x-x^{\prime}\right)^{2}\right]\right\} d x^{\prime}
$$

Here $A_{0}$ is the amplitude of an incident plane wave, $k=2 \pi / \lambda$ is its wave number, $z$ is a distance between the phase screen and the ground, $x$ is the position coordinate, and $\phi(x)=-\lambda r_{e} \Delta N_{T}(x)$ is a phase change of the incident wave on the screen due to the deviation of the total electron content $\Delta N_{T}$ through the irregularity slab $\left(r_{e}=\right.$ classical electron radius). Equation (1) can be integrated using the Fourier transformation, provided the phase fluctuations $\phi(x)$ are known.

To model the phase fluctuations, it has been assumed that they represent a Gaussian random process with a power spectrum given by

$$
S_{\phi}(\kappa)=C\left(\kappa_{0}^{2}+\kappa^{2}\right)^{-p / 2}
$$

The structure strength parameter $C$ in (2) is

$$
C=2 \lambda^{2} r_{e}^{2} \sigma_{N}^{2} L \kappa_{0}^{p-2} \frac{\Gamma(p / 2)}{\Gamma((p-2) / 2)}
$$

where $\sigma_{N}^{2}$ is the electron density variance, $L$ is the irregularity slab thickness, and $\kappa_{0}=2 \pi / r_{0}\left(r_{0}=\right.$ outer scale). The spectral index $p$ is 1 less than the spectral index for a three-dimensional electron density fluctuations.

The phase field $\phi(x)$ has been generated by taking the inverse Fourier transform of $2\left[S_{\phi}(\kappa) \Delta \kappa\right]^{1 / 2}$ multiplied by phase angles uniformly distributed over the interval $-\pi, \pi$ [cf. Rice, 1954] with $\Delta \kappa=2 \pi / \Delta x$, where $\Delta x$ is the step size along $x$.

In the actual numerical calculations, $\phi(x)$ was generated for various spectral indices $p$ and fixed values of $r_{0}, L, z$, and $\Delta x$ equal to $15 \mathrm{~km}, 100 \mathrm{~km}$, $350 \mathrm{~km}$, and $21 \mathrm{~m}$, respectively, and $\sigma_{N}^{2}$ was chosen to cover three regimes of scintillation: weak, moderate, and strong. The wavelength $\lambda$ is $2.2 \mathrm{~m}$ corresponding to the beacon wave frequency $137 \mathrm{MHz}$ used in the experiment described later on.

Figure 1 shows an example of a random phase field (upper panel) generated for $p=2.35$ and corresponding amplitude scintillation calculated using (1) for three regimes of scintillation. To compare with observations the spatial coordinate $x$ has been converted to time assuming the speed of the diffraction pattern $\nu=1.5 \mathrm{~km} / \mathrm{s}$.

\section{Reconstruction of Information Dimension}

Before proceeding further, we will make an important remark. The phase fluctuations $\phi(x)$, as 

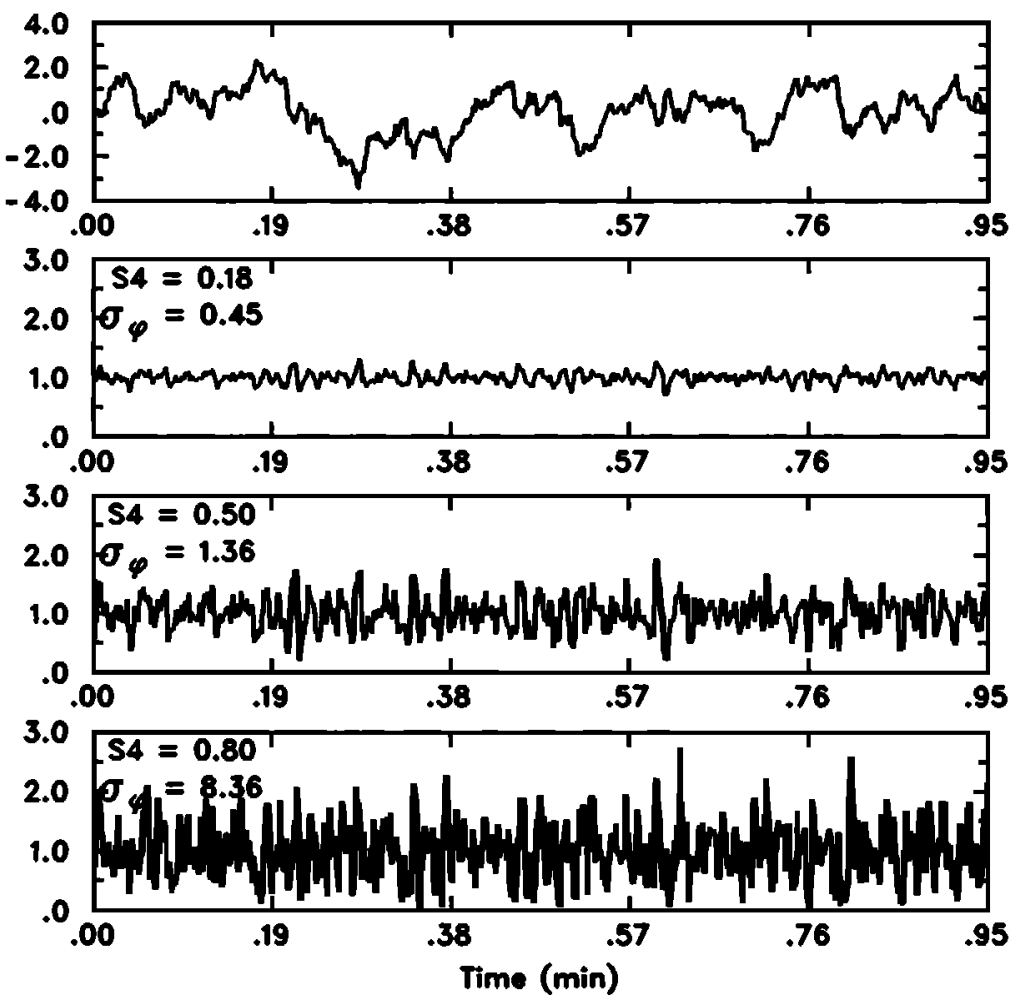

Figure 1. An example of simulated random phase screen (upper panel) and amplitude scintillation patterns for three levels of scintillation intensity as quantified by the scintillation index $S_{4}$ and rms phase $\sigma_{\phi}$. Spectral index $p=2.35$.

generated in our model, represent a random colored noise and not a deterministic chaos with the same form of spectrum and well-defined attractor. As has been demonstrated by Osborne and Provenzale [1989] and will become evident also from our calculations, such a noise has a finite information dimension. This means that the colored noise has fractal properties which entitles us to use notions taken from the chaos theory. An often used method of distinguishing between stochastic processes and deterministic chaos is based on the fact that the Fourier components of random noise are not correlated. In this method the information dimension analysis is performed twice: on the original data and substitute data. Substitute data are obtained by (1) taking the Fourier transform of the original data, (2) introducing random phases, and (3) taking the inverse Fourier transform. If the information dimension for substitute data is the same as that for original data, then the original data set represents random noise.

The kind of data shown in Figure 1 has been used to reconstruct the information dimension for random phase on a screen and scintillation on the ground. The first step in the procedure is the reconstruction of the attractor in the phase space and, second, finding the dimension of the attractor.

To reconstruct the attractor from the discretely sampled time series $\{x(t)\}(t=0, \Delta t, 2 \Delta t, \cdots, N \Delta t)$ the classical method of time delay coordinates is most often used [Packard et al., 1980; Takens, 1981]. In this method a vector $X_{i}$ in an $m$-dimensional phase space is constructed by taking delayed samples of the time series as coordinates:

$$
\mathbf{X}_{i}=\left(x\left(t_{i}\right), x\left(t_{i}+\tau\right) \cdots x\left(t_{i}+(m-1) \cdot \tau\right)\right)
$$

where $\tau$ is a fixed time delay equal to the multiple $n$ of the sampling time $\Delta t$ and $t_{i}$ is the discrete time.

It has been shown [Takens, 1981] that in order to reconstruct the attractor of dimension $d$ the embedding dimension $m$ must be at least equal to $2 d+1$. For a long series of the noise-free data an arbitrary time delay $\tau$ (hence $n$ ) can be taken. In practice, 

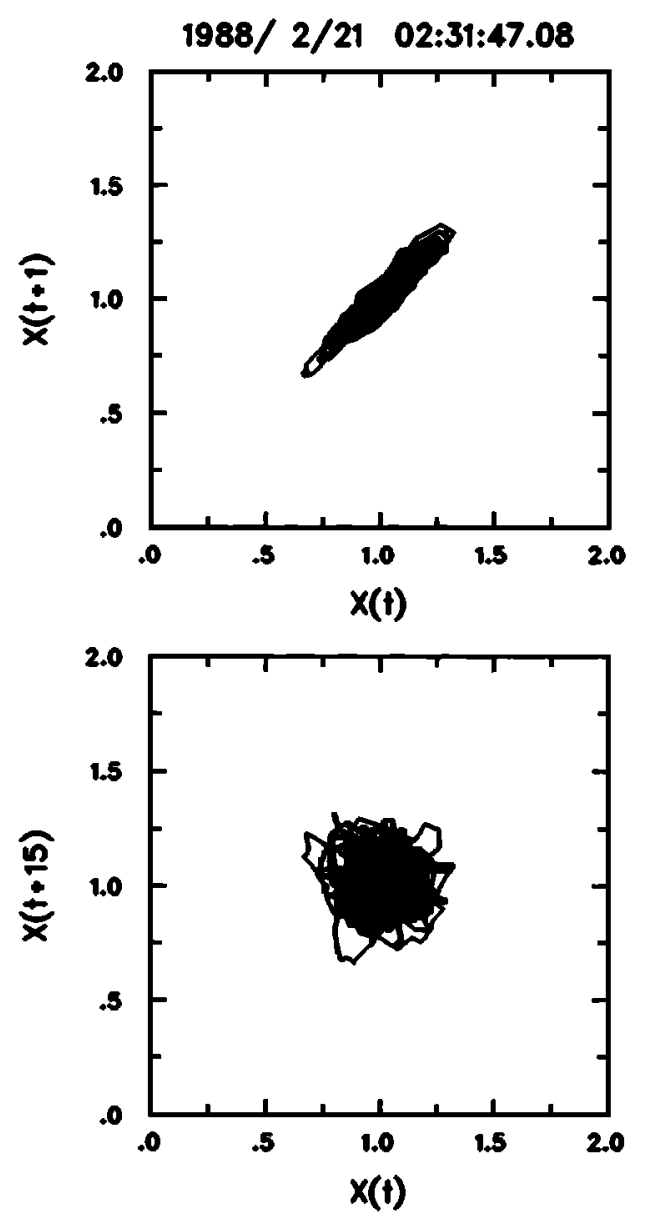

Figure 2. An example of the two-dimensional phase portrait of measured amplitude scintillation for two delays showing stretching of the attractor when the delay is too small.

however, the choice of $\tau$ is critical since the coordinates of the reconstructed attractor must be as statistically independent as possible. Fraser and Swinney [1986] have presented a method to find the optimum value of $\tau$ by calculating the mutual information. The method is complicated and requires very large data sets unless the dimension is very low. Instead, as the first trial we have used the time delay corresponding to the first zero of the autocorrelation function and then tried smaller and larger delays looking for the dependence of the information dimension on $\tau$. It is obvious that the information dimension should not depend on the delay.

To demonstrate the effect of time delay on the attractor reconstruction, we show in Figure 2 the two-dimensional phase portraits constructed for one of our experimental data sets for $n=1$ and $n=$ 15. This latter value of $n$ corresponds to the first zero of the autocorrelation function. One can see that due to a high degree of correlation between points in the phase space the attractor for $n=1$ is stretched along the diagonal which apparently will give an underestimated attractor dimension.

In order to test the choice of $\tau$ we have used a phase space construction procedure adopted from Osborne and Provenzale [1989]. Rather than using a single time series, 15 independent time series representing the phase fluctuations on the screen have been generated. The point in $m$-dimensional space is represented by (3) but each coordinate $x\left(t_{i}+(m\right.$ - 1) $\cdot \Delta t)(m=1, \cdots, 15)$ is now taken from different realizations. This procedure assures the independence of closely spaced points in the phase space. The information dimension is then computed by the method described in the next section and compared with that for a single series. It has been found that indeed the choice of $\tau$ is important and that the optimal delay has a value as large as compared to the correlation time, possibly close to the first zero of the autocorrelation function.

The most popular method of the attractor dimension reconstruction is that proposed by Grassberger and Procaccia [1983]. However, it has been shown [Holzfuss and Mayer-Kress, 1989] that the so-called pointwise dimension method [Mandelbrot, 1983] gives a smaller error in the calculated dimension, especially for high-dimensional attractors. The method consists in counting the number of points $N_{\mathbf{X}_{0}}(r)$ within a sphere of radius $r$ centered at a certain $\mathbf{X}_{0}$ point in the phase space and computing the dimension $D_{I}$ from

$$
D_{I}=\lim _{r \rightarrow 0} \frac{\ln N_{\mathbf{X}_{0}}(r)}{\ln r}
$$

If $D_{I}$ is computed for a number of embedding dimensions $m$, then for sufficiently large $m, D_{I}$ should become independent of $m$. This constant value of $D_{I}$ is a measure (lower bound) of the fractal dimension $d$.

In practice, it is necessary to take many randomly chosen reference points $\mathbf{X}_{0}$ and compute the average to get a reliable estimate of the attractor dimension. We experimented with several numbers of the reference points $N_{\text {ref }}$ and found that a good compromise between the error in $D_{I}$ and computation efficiency is reached when $N_{\text {ref }}=50$. It should be 
mentioned that when computing the distance between points we used the Euclidan norm as recommended by Havstad and Ehlers [1989].

The crucial point is the choice of the range of $r$ for which $D_{I}$ is calculated. By definition, $D_{I}$ in (4) is estimated from the $\ln N-\ln r$ plot by fitting a straight line at small $r$. Unfortunately, that is the region of $r$ sensitive to various distortions [Havstad and Ehlers, 1989; Caputo et al., 1989]. The most dangerous distortion is caused by oversampling which generates large number of vectors along a short segment of the trajectory in the phase space producing a tail of slope 1 at small $r$. Apparently, if not eliminated, this distortion will underestimate the dimension. For this reason, in computing $D_{I}$ the $\ln N-\ln r$ plot is inspected for the presence of a tail. The data are decimated until the tail disappears. Unfortunately, this procedure reduces the number of data points.

Another distortion reducing the useful range of $r$ results from the fact that number of points within a specific volume of phase space have a Poisson distribution which requires a modification of (4) [Havstad and Ehlers, 1989]:

$$
D_{I}=\lim _{r \rightarrow 0} \frac{\psi\left(N_{\mathbf{x}_{0}}(r)\right)}{\ln r}
$$

where $\psi(x)=d(\ln \Gamma(x)) / d x$ is the digamma function. This modification stretches the ordinate at small

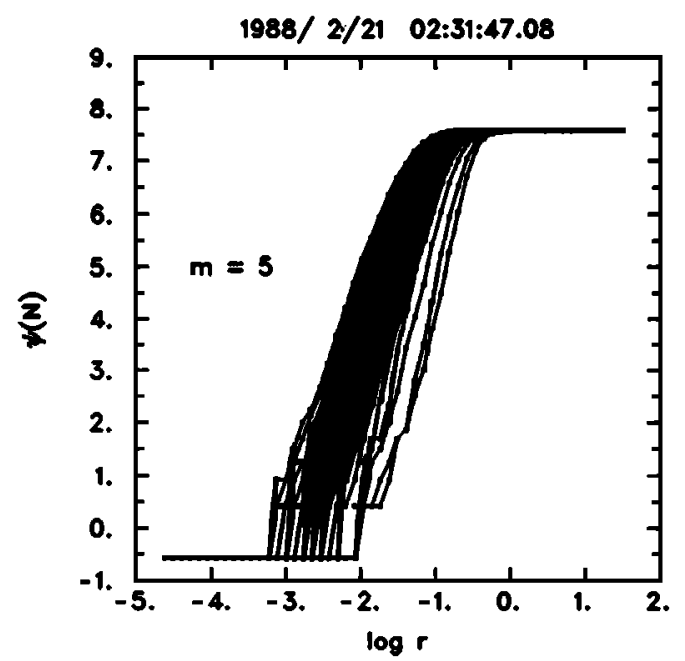

Figure 3. The $\psi(N)$ versus $\log r$ curves for actually measured amplitude scintillation. Computations were made for the embedding dimension $m=5$ and 50 reference points.

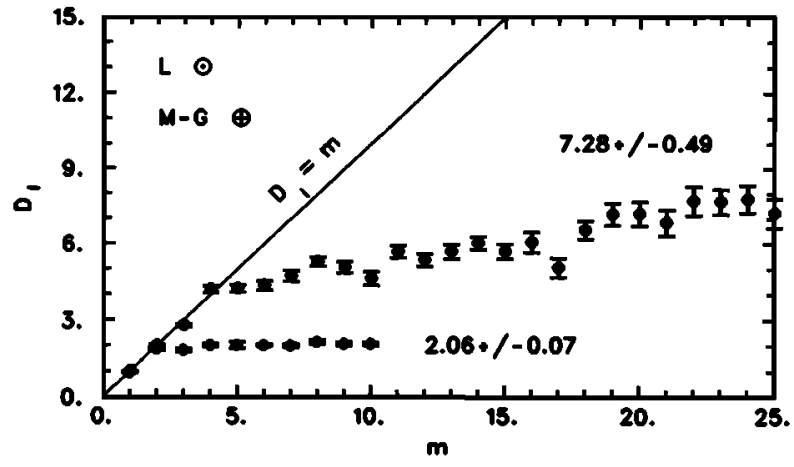

Figure 4. $D_{I}(m)$ for the Lorenz (open circles) and Mackey-Glass (crossed circles) attractors. The rms errors are also shown.

values of $N$, resulting in an increased slope of the curve. An example of the $\psi(N)-\ln r$ plot for actually measured scintillation is given in Figure 3 for $N_{\text {ref }}=50$ and the embedding dimension $m=5$.

The program evaluating $D_{I}$ has been designed for a full automatic analysis of data. A straight line is fitted by least squares to a certain segment of $\psi-\ln$ $r$ curve using the FIT routine of Press et al. [1988]. Both the length of the segment and its starting point are varied and the $\chi^{2}$ goodness of fit test is used to choose the best scaling range of $r$. The average slope is calculated with weights proportional to the variances of each individual slope. Only those reference points are used for which the goodness of fit parameter is larger than 0.5 . The procedure has been verified for the Lorenz and Mackey-Glass attractors for which the dimensions are known from other sources. The Lorenz attractor is modeled by solving the well-known set of equations:

$$
\begin{aligned}
& \dot{X}=16.0(Y-X) \\
& \dot{Y}=X(45.92-Z)-Y \\
& \dot{Z}=X Y-4.0 Z
\end{aligned}
$$

The $D_{I}$ versus $m$ plot is shown in Figure 4. One can see that $D_{I}$ tends to a constant value for $m$ sufficiently large. The information dimension calculated as the average of $D_{I}$ for $m \geq 6$ is equal $2.06 \mp$ 0.07 , which agrees with the value 2.07 calculated from the Lyapunov exponents [Wolf et al., 1985].

Since specific problems might be encountered when reconstructing high-dimensional attractors, we have tested the procedure also for the MackeyGlass attractor [Mackey and Glass, 1977] which is 


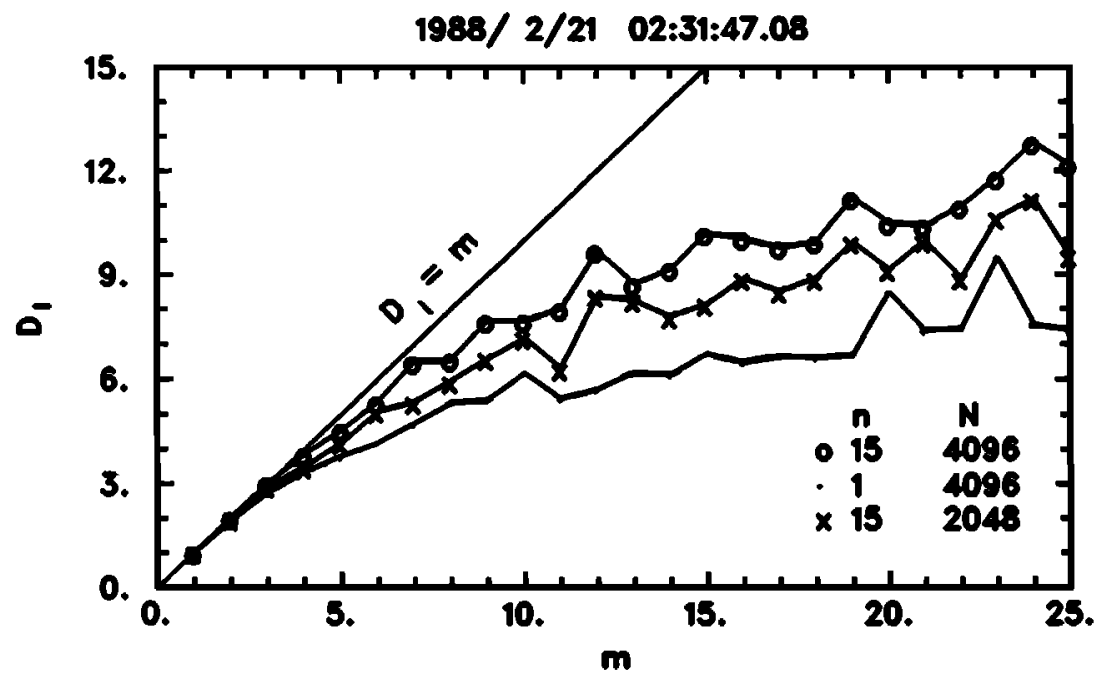

Figure 5. $D_{I}(m)$ for the measured amplitude scintillation showing the effect of the delay and sample length on the estimate of the attractor dimension.

known to have a high dimension. The MackeyGlass equation

$$
\dot{x}=\frac{0.2 x(t-\tau)}{1+[x(t-\tau)]^{10}}-0.1 x(t)
$$

with the delay parameter $\tau=100$ has been solved using the method similar to that described by Havstad and Ehlers [1989]. The plot $D_{I}(m)$ is shown in Figure 4. The information dimension calculated as the average $D_{I}$ for $m \geq 18$ is equal to $7.28 \mp 0.49$, which is smaller but close to the correct value 7.5 [Havstad and Ehlers, 1989]. The difference might be caused by too small a data set having been taken for the analysis. We will discuss briefly the effect of data set length on the dimension estimate.

It is generally accepted that for larger data sets the dimension estimate is more reliable. However, specialists in chaotic system analysis have not reached a consensus as to what is the smallest useful data set. For instance, Ruelle [1990] claims that if the dimension is determined from a data set of length $N$ over at least one decade in $r$, then one should not believe dimension estimates that are not well below $2 \log _{10} N$. Thus the maximum slope for $N=4096$, which is the number of points used in our analysis, is approximately 7. Roberts [1991] argues that $N>10^{d}$ is required. It seems that this is too pessimistic an estimate. Havstad and Ehlers [1989] have shown by numerical computations that the independence of points in the phase space is essen- tial and, if it is satisfied, even a small data set would give a reliable estimate of the dimension.

In view of such differing opinions we have decided to experiment with several data sets of different lengths. An example is given in Figure 5 where $D_{I}(m)$ is plotted for $N=4096$ and 2048 for one of our experimental data sets. It is seen that for smaller $N, D_{I}$ is smaller. Since we can not increase $N$ over 4096 due to the danger of nonstationarity we conclude that in this particular case the information dimension is higher than 12 . In the same figure the already discussed effect of time delay is illustrated. One can see that for $n=1, D_{I}$ is much smaller than for $n=15$, which is the zero autocorrelation delay for this data set.

\section{Chaotic Behavior of Simulated Scintillation}

The method described in the previous section has been used to calculate the information dimensions of the modeled phase fluctuations on a screen as well as simulated amplitude and phase scintillation of the diffracted wave. The aim of these calculations is to look for a relationship between the chaotic behavior of scintillation and ionospheric irregularities, if any.

Figure 6 shows $D_{I}(m)$ for random phase fluctuations on a screen (representing the ionosphere) for three values of the spectral index $p$. One can note the saturation of $D_{I}$ for large embedding dimensions 


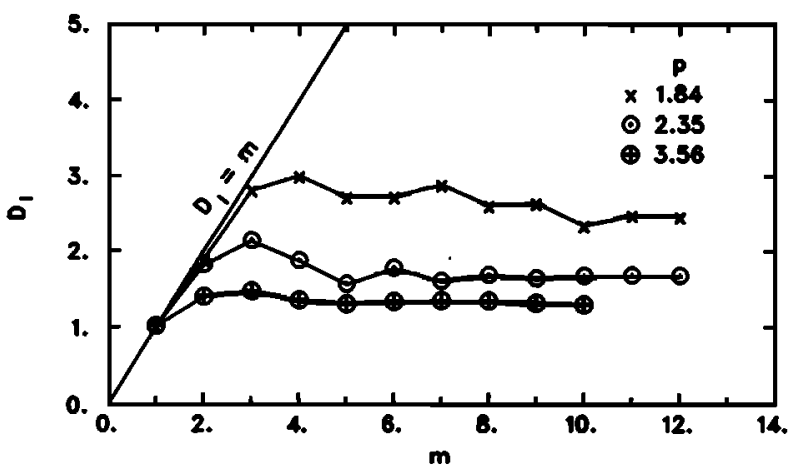

Figure 6. $D_{I}(m)$ for random fluctuations on the phase screen characterized by the spectral index $p$.

which means that the information dimension is finite and can be determined from the plot. In Figure 7 we plotted the dimension of the phase attractor as a function of the spectral index $p$. The dimension falls rapidly with increasing $p$, approaching the asymptotic value of 1 . This result agrees with more detailed calculations by Osborne and Provenzale [1989] for colored random noise, similar to our random phase fluctuations.

Figure 8 presents the behavior of $D_{I}$ for simulated scintillation. Upper panel corresponds to $p=1.84$, and the lower one to $p=2.35$. For each $p$, three curves are plotted representing three levels of scintillation intensity, as measured by the scintillation index $S_{4}$. For other values of $p$ the behavior of $D_{I}(m)$ is very similar. A surprising result is that $D_{I}$ does not saturate even at such high embedding dimension as 20 , which means that we cannot claim

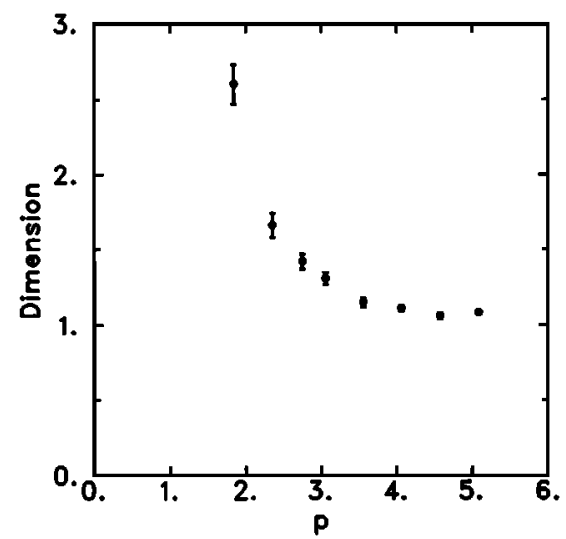

Figure 7. The dependence of information dimension of random fluctuations on the phase screen on the spectral index $p$.
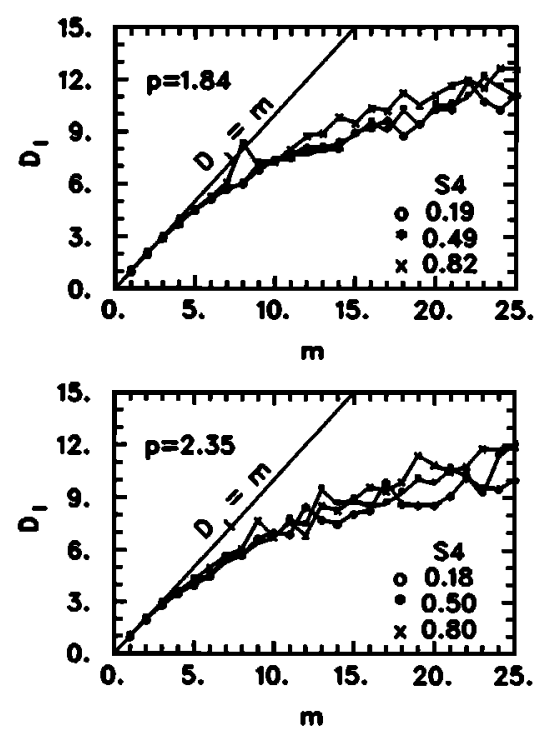

Figure 8. Examples of $D_{I}(m)$ for modeled amplitude scintillation corresponding to two spectral indices $p$. Three curves are drawn for weak, moderate, and strong scintillation.

the existence of the scintillation attractor and, if it exists, its dimension is considerably higher than 12 . Another interesting feature is the independence of $D_{I}(m)$ curves on the scintillation intensity, at least over the range of $m$ considered here. Note that in spite of a strong dependence of the dimension of phase fluctuation attractor on $p$ (cf. Figure 7), for scintillation the dependence of $D_{I}$ on $p$ is very weak. Only with some effort can one see that the larger the spectral index $p$, that is, the steeper the spectra, the smaller are $D_{I}$ values at large embedding dimensions, reflecting the dependence of the scintillation attractor dimension on the phase fluctuations attractor. The effect is, however, very small and certainly not strong enough to be found in experimental data. The conclusion is that (provided the assumptions made in the scintillation simulations are satisfied) the amplitude scintillation attractor, if it exists, reflects the propagation effects rather then the chaotic behavior of irregular ionosphere and that from studying the amplitude scintillation attractor alone, no additional information about the irregularities can be gained.

The information dimension has also been computed for simulated phase scintillations on the ground and compared with that for the phase screen. An example of comparison is shown in Figure 9 for the phase spectrum with $p=2.35$ and 


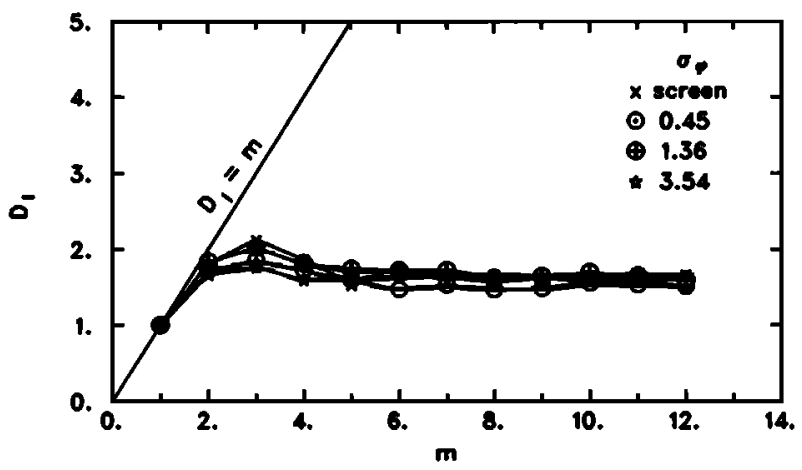

Figure 9. $D_{I}(m)$ for random fluctuations on the phase screen and modeled phase scintillation on the ground with various rms values of phase $\sigma_{\phi}$.

three values of rms phase $\sigma_{\phi}$ corresponding to the weak, moderate, and strong amplitude scintillation. One can see that the asymptotes of all $D_{I}(m)$ curves agree which means that the phase scintillation has the same information dimension as ionospheric irregularities independent of $\sigma_{\phi}$; thus the phase scintillation measurements can be used to reproduce the fractal structure of ionospheric turbulence.

\section{Chaotic Behavior of Measured Scintillation}

To compare the results of modeling with observations we have used amplitude scintillation of Hilat and Polar Bear 137-MHz beacon signals recorded at the Polish Polar Station at Hornsund, Svalbard. The details of setup and reduction method are described elsewhere [Wernik et al., 1990]. Here we only mention a few important features. Original data are recorded on an FM analogue tape recorder, then digitized at a rate approximately 250 samples per second. A low-pass, sixpole Butterworth filter with a cutoff at $0.1 \mathrm{~Hz}$ is used to detrend the data. Each raw value of received power is divided by the corresponding value of the trend. Since it has been found that spectral intensities reach the noise floor at frequencies slightly higher than $30 \mathrm{~Hz}$, detrended data are decimated by a factor of 4 after appropriate lowpass filtering needed to eliminate aliasing. The resulting Nyquist frequency is $35.89 \mathrm{~Hz}$.

Three sets of data corresponding to the weak, moderate, and strong scintillation have been analyzed. In Figure 10 we show their $D_{I}(m)$ plots. In all three cases a characteristic feature of $D_{I}(m)$ curves
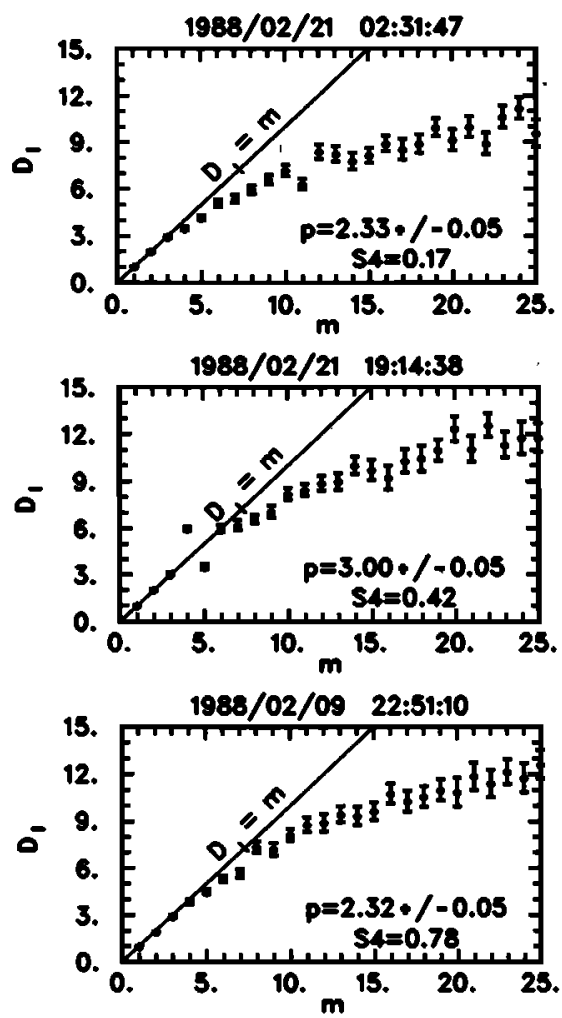

Figure 10. Examples of $D_{l}(m)$ for measured weak, moderate, and strong amplitude scintillation as quantified by the scintillation index $S_{4}$.

is a lack of saturation and high values of $D_{I}$ at large $m$. This behavior of $D_{I}(m)$ seems to be independent of the scintillation intensity and spectral index. The agreement with the results of simulation is striking. Unfortunately, we have no phase scintillation measurements at our disposal; thus its comparison needs to be deferred to the future.

\section{Discussion and Conclusions}

In this paper we tried to relate the chaotic behavior of ionospheric turbulence to the amplitude and phase scintillations. On the basis of the simulation calculations, we conclude that the propagation effects would mask the effects of turbulence, and thus the ionospheric turbulence attractor cannot be reconstructed from the amplitude scintillation analysis. This conclusion agrees with Bhattacharyya's [1990] results. However, at one point our results disagree with that of Bhattacharyya. She has found an increase of $D_{I}$ with the scintillation intensity, while both simulation and data analysis presented 
here show that it is independent of scintillation intensity. This disagreement might be due to the fact that in all her cases, Bhattacharyya used the delay time $\tau$ equal to the sampling time $\Delta t$. We have shown that the optimal delay is that equal to the first zero of the autocorrelation function $\tau_{o}$; otherwise, the attractor dimension is underestimated. For small scintillation intensity $\tau_{o}$ might be much larger than the sampling time, and consequently, $D_{I}$ will be underestimated. Additionally, $D_{I}$ is underestimated because the data sets analyzed by Bhattacharyya are very small $(N=800)$. With increasing scintillation intensity, $\tau_{o}$ decreases, becoming closer to $\Delta t$, and the dimension estimate increases.

In spite of a failure of amplitude scintillation analysis to reproduce the chaotic behavior of ionospheric turbulence, the analysis of phase scintillation measurements could be valuable in studying the chaos in the ionosphere.

The difference in the chaotic behavior of amplitude and phase scintillation is apparently related to the physical mechanisms responsible for each type of scintillation [Yeh and Liu, 1982]. As the wave propagates inside the irregularity slab, to the first order, only its phase is affected by the random fluctuations of refractive index and when emerging from the slab, the wave front is phase modulated. As the wave propagates a distance $z$ below the slab an interference pattern develops, resulting in amplitude fluctuations. The major contribution to the amplitude scintillation comes from irregularities smaller than the size of the first Fresnel zone $F \approx$ $(\lambda z)^{1 / 2}$. When the rms phase fluctuation $\sigma_{\phi}$ at the exit from the slab is small, the phase mixing results in a complicated, random interference pattern on the ground, very much different from the irregular structure of the slab. Simulations show that even very close to the phase screen the amplitude scintillation pattern does not resemble the phase pattern on a screen. Because of that, the chaotic behavior of irregular ionosphere cannot be reconstructed from amplitude scintillation. For small $\sigma_{\phi}$, only lower-order moments of scintillation (scintillation index, correlation function, and power spectrum) can be related to the moments of random fluctuations of electron density. When the rms phase fluctuation $\sigma_{\phi}$ exceeds $1 \mathrm{rad}$, irregularities larger than $F$ also contribute to the amplitude scintillation causing focusing and defocusing of individual rays. However, this effect would not change already complicated, random amplitude scintillation pattern on the ground. On the other hand, the phase fluctuations at the bottom of the irregular slab map directly onto the ground without too much distortion caused by the interference. Thus the chaotic behavior of phase scintillation can be directly related to the chaotic behavior of the ionosphere.

In our discussion of the information dimension reconstruction methods we stressed that the analysis must be made with the proper account for effects which affect the information dimension estimate and could lead to erroneous results. The most important effects are those related to the choice of length of the data set and of the delay time.

Acknowledgments. We thank the crew of the Polish Polar Station at Svalbard for their help with the observations. The station is operated by the Institute of Geophysics of the Polish Academy of Sciences. The joint work is supported by the National Science Foundation under INT 92-00765. The work by A.W.W. has been supported in part by the Polish Committee on Scientific Research under the grant $221509203 \mathrm{p} / 31$.

\section{References}

Bergé, P., Y. Pomeau, and C. Vidal, Order Within Chaos, John Wiley, New York, 1987.

Bhattacharyya, A., Chaotic behavior of ionospheric turbulence from scintillation measurements, Geophys. Res. Lett., 17, 733-736, 1990.

Caputo, J. G., B. Malraison, and P. Atten, Determination of attractor dimension and entropy for various flows: An experimentalist's view point, in Dimensions and Entropies in Chaotic Systems, edited by G. MayerKress, pp. 180-190, (2nd corrected printing), Springer Verlag, New York, 1989.

Costa, E., and M. C. Kelley, On the role of steepened structures and drift waves in equatorial spread F, $J$. Geophys. Res., 83, 4359-4364, 1978.

Fraser, A. M., and H. L. Swinney, Independent coordinates for strange attractors from mutual information, Phys. Rev. A, 33, 1134-1140, 1986.

Grassberger, P., and I. Procaccia, Characterization of strange attractors, Phys. Rev. Lett., 50, 346-349, 1983.

Hassam, A. B., W. Hall, J. D. Huba, and M. J. Keskinen, Spectral characteristics of interchange turbulence, $J$. Geophys. Res., 91, 13,513-13,522, 1986.

Havstad, J. W., and C. L. Ehlers, Attractor dimension of nonstationary dynamical systems from small data sets, Phys. Rev. A, 39, 845-853, 1989.

Holzfuss, J., and G. Mayer-Kress, An approach to errorestimation in the application of dimension algorithms, in Dimensions and Entropies in Chaotic Systems, edited by G. Mayer-Kress, pp. 114-122, (2nd corrected printing), Springer Verlag, New York, 1989. 
Huba, J. D., A. B. Hassam, I. B. Schwartz, and M. J. Keskinen, Ionospheric turbulence: Interchange instabilities and chaotic fluid behavior, Geophys. Res. Lett., $12,65-68,1985$.

Knepp, D. L., Multiple phase-screen calculation of the temporal behavior of stochastic waves, Proc. IEEE, 71, 722-737, 1982.

Mackey, M. C., and L. Glass, Oscillation and chaos in physiological control systems, Science, 197, 287, 1977.

Mandelbrot, B., The Fractal Geometry of Nature, W. H. Freeman, New York, 1983.

Osborne, A. R., and A. Provenzale, Finite correlation dimension for stochastic systems with power-law spectra, Phys. D Amsterdam, 35, 357-381, 1989.

Packard, N. H., J. P. Crutchfield, J. D. Farmer, and R. S. Shaw, Geometry from a time series, Phys. Rev. Lett., 45, 712-716, 1980.

Press, W. H., B. P. Flannery, S. A. Teukolsky, and W. T. Vetterling, Numerical Recipes, The Art of Scientific Computing, Cambridge University Press, New York, 1988.

Rice, S. O., Mathematical analysis of random noise, in Selected Papers on Noise and Stochastic Processes, edited by N. Wax, Dover, Mineola, New York, 1954.

Rino, C. L., and J. Owen, Numerical simulations of intensity scintillation using the power law phase screen model, Radio Sci., 19(3), 891-908, 1984.
Roberts, D. A., Is there a strange attractor in the magnetosphere?, J. Geophys. Res., 96(A9), 16,031-16,046, 1991.

Takens, F., Detecting strange attractors in turbulence, in Dynamical Systems and Turbulence, Lecture Notes in Mathematics, vol. 898, edited by D. A. Rand and L. S. Young, pp. 366-381, Springer, New York, 1981.

Wernik, A. W., C. H. Liu, S. J. Franke, and M. Gola, High-latitude irregularity spectra deduced from scintillation measurements, Radio Sci., 25, 883-895, 1990.

Wolf, A., J. Swift, H. L. Swinney, and J. A. Vastano, Determining Lyapunov exponents from a time series, Phys. D Amsterdam, 16, 285-317, 1985.

Yeh, K. C., and C. L. Liu, Radio wave scintillations in the ionosphere, Proc. IEEE, 70, 324-360, 1982.

A. W. Wernik, Space Research Centre, Polish Academy of Sciences, ul. Bartycka 18a, 00-716 Warsaw, Poland.

K. C. Yeh, College of Engineering, National Sun YatSen University, Kaohsiung, Taiwan 80424 Republic of China.

(Received January 8, 1993; revised June 29, 1993;

accepted June 29, 1993.) 